\title{
Covid-19: Cycle of 50 day lockdowns and 30 day relaxations could be effective, study finds
}

\author{
Gareth lacobucci
}

The BMJ

An alternating cycle of 50 days of lockdown followed by 30 days of easing could be an effective strategy for reducing deaths and intensive care admissions from covid-19, an international modelling study has shown. ${ }^{1}$

But this would need to be accompanied by efficient testing, case isolation, contact tracing, and shielding of vulnerable people, said researchers from the Global Dynamic Interventions Strategies for Covid-19 Collaborative Group.

The study, published in the European Journal of Epidemiology, assessed the likely impact of alternating between stricter measures such as lockdown and intervals of more relaxed social distancing, amid concerns that lockdowns may be unsustainable over long periods, given their economic and social impact.

It modelled three scenarios across 16 economically diverse countries, comparing less strict "mitigation" strategies (case based isolation, shielding vulnerable groups, school closures, and restricting public events) with "suppression" strategies (strict physical distancing, including lockdown) and following each with a relaxation period (case based home isolation of symptomatic cases and shielding vulnerable groups).

Rajiv Chowdhury, global health epidemiologist at the University of Cambridge and lead author on the paper, said, "Our models predict that dynamic cycles of 50 day suppression followed by a 30 day relaxation are effective at lowering the number of deaths significantly for all countries throughout the 18 month period.

"This intermittent combination of strict social distancing, and a relatively relaxed period with efficient testing, case isolation, contact tracing, and shielding the vulnerable, may allow populations and their national economies to 'breathe' at intervals - a potential that might make this solution more sustainable, especially in resource poor regions."

\section{Scenarios}

The UK was not among the countries modelled, but Chowdhury said that the effects would probably be very similar to those projected in other high income countries.

In the first scenario, which modelled the impact of imposing no measures, the number of patients requiring treatment in intensive care units (ICUs) would quickly exceed the available capacity considerably in all 16 countries, resulting in 7.8 million deaths in total, and the epidemic would last nearly 200 days in most countries, the researchers found.
The second scenario modelled a rolling cycle of 50 day mitigation measures followed by a 30 day relaxing. Such a strategy would probably reduce the R number (the number of people that each infected individual goes on to infect) to 0.8 in all countries. But it suggested that, after the first relaxation, the number of patients requiring ICU care would exceed hospital capacity and result in 3.5 million deaths throughout the 16 countries. In this scenario the pandemic would last about 12 months in high income countries and 18 months or longer in the other settings, the study estimated.

The final scenario was a rolling cycle of stricter 50 day suppression measures followed by a 30 day relaxing. This strategy would reduce the R number to 0.5 and keep ICU demand within national capacity in all countries. It would result in a longer pandemic, exceeding 18 months in all countries, but considerably fewer people would die during that period-just over 130000 throughout the 16 countries, the researchers estimated.

\section{Practicality}

Oscar Franco, from the University of Bern in Switzerland and coauthor of the paper, said, "There's no simple answer to the question of which strategy to choose. Countries-particularly low income countries-will have to weigh up the dilemma of preventing covid-19 related deaths and public health system failure with the long term economic collapse and hardship."

The research was funded by the European Union's Horizon 2020 programme.

Gabriel Scally, visiting professor of public health at the University of Bristol, UK, and a member of the Independent SAGE group, said that 50/30 day alternating could be impractical. "Theoretically it could work, but not practically, because I don't think businesses can scale up and scale down like that," he told The BMJ.

Scally suggested that a more effective approach would be getting the number of infections much lower through testing and contact tracing, while reserving severe social distancing for hotspot areas. "This study seems to be based on a universalist approach where the whole country shuts down, whereas I think we're moving into a phase now where we need differential approaches [in different geographical areas]," he said.

Chowdhury R, Heng K, Shawon SR, etal . Dynamic interventions to control covid-19 pandemic: a multivariate prediction modelling study comparing 16 worldwide countries. Eur J Epidemiol 2020. 10.1007/s10654-020-00649-w. 
